\title{
The effect of red yeast rice on serum and haemodynamic risk factors for cardiovascular disease
}

\author{
F. E. Lithander ${ }^{1}$ and A. Mahmud ${ }^{2}$ \\ ${ }^{1}$ Discipline of Nutrition and Dietetics, The University of Canberra, ACT, Australia and ${ }^{2}$ Department of General \\ Medicine, Royal College of Surgeons in Ireland, Medical University of Bahrain, Kingdom of Bahrain
}

Chinese red yeast rice (RYR) is a dietary supplement containing monacolin, a naturally occurring hydroxymethylglutaryl-CoA reductase (HMG-CoA reductase) inhibitor. Several studies have demonstrated that RYR can decrease serum markers of cardiovascular disease (CVD) such as total cholesterol (TC) ${ }^{(1)}$ and low density lipoprotein cholesterol (LDL-C) ${ }^{(2)}$. The aim of the current study was to investigate the effect of RYR on such markers, in addition to non-serum markers of CVD risk such as arterial stiffness.

Twenty four lean, healthy participants (age 22, 1y (mean, SD)) took part in this randomised, placebo-controlled, double-blind study. Each participant was randomised to one of two groups for a period of 28 days; RYR $600 \mathrm{mg} / \mathrm{d}$ or placebo, which comprised of ground white rice in matching capsules. Participants were asked to consume the capsules each night for the intervention period. Fasted blood samples were collected, and blood pressure and arterial stiffness [pulse wave velocity (PWV) and augmentation index (AIx)] were measured at baseline and on day 28 .

At baseline, all participants had normal lipid profile, plasma glucose and hs-CRP $<3.0 \mathrm{mg} / \mathrm{dl}$, and there was no significant difference in these parameters between the two groups $(P>0 \cdot 05)$. All haemodynamic measures were normal at baseline, and there was no significant difference in brachial and aortic blood pressure, PWV or AIx between the two groups $(P>0.05)$ at baseline. On day 28 , there was a significant reduction in total TC $(P<0.001)$, LDL-C and hs-CRP with RYR $(P<0.01)$ compared with placebo. There was no significant difference in HDL-C, triglycerides or plasma glucose with either RYR or placebo at the end of the intervention period. There was no significant change in brachial or aortic blood pressure, mean arterial pressure (MAP) or heart rate over the 28d period. However, there was a significant reduction in PWV with RYR $(P<0.01)$. There was no change in AIx with either treatment.

This study has demonstrated that monacolin, when consumed in RYR, has a beneficial effect on both serum and non-serum markers of CVD risk. The study highlights the need for further research over longer periods of time to investigate the effects of such a dietary supplement on risk factors for chronic disease in different patient groups.

1. Cicero AF, Derosa G, Parinia A et al. (2013) Nutr Res 33, 622-628.

2. Verhoeven V, Hartmann ML, Remmen R et al. (2013) BMC Compl Alt Med, 13:178-186. 